Int. J. Dev. Biol. 48: 149-156 (2004)

\title{
Drm/Gremlin, a BMP antagonist, defines the interbud region during feather development
}

\author{
BORIS BARDOT ${ }^{\#, \alpha, 1}$, LAURE LECOIN,1, INGRID FLINIAUX², EMMANUELLE HUILLARD ${ }^{1}$, \\ MARIA MARX ${ }^{1}$ and JEAN P. VIALLET ${ }^{*, 2}$ \\ ${ }^{1}$ Régulations Cellulaires et Oncogenèse, UMR146 du CNRS, Institut Curie, Centre Universitaire, Orsay \\ and ${ }^{2}$ LEDAC, UMR 5538 du CNRS, Institut A. Bonniot, Université. J. Fourier, Grenoble, France
}

\begin{abstract}
The pattern of feather buds in a tract is thought to result from the relative ratios between activator and inhibitor signals through a lateral inhibition process. We analyse the role of Drm/Gremlin, a BMPs antagonist expressed during feather pattern formation, in the dermal precursor, the dense dermis, the interbud dermis and in the posterior dermal condensation. We have altered the activity of Drm in embryonic chick skin using retroviral vectors expressing $\mathrm{drm} /$ gremlin and bmps. We show that expression of endogenous drm is under the control of a feedback loop induced by the BMP pathway, and that overexpression of drm results in fusion between adjacent feather buds. We propose that endogenous BMP proteins induce drm expression in the interbud dermis. In turn, the Drm/Gremlin protein limits the inhibitory effect of BMPs, allowing the adjacent row of feathers to form. Thus, the balance between BMPs and its antagonist Drm would regulate the size and spacing of the buds.
\end{abstract}

KEY WORDS: drm/gremlin, BMP, skin, feather bud

\section{Introduction}

The avian embryonic skin is a classical experimental model of pattern formation. During development, feathers are generated following a sequentially reiterated process at defined positions within the embryo, alternating feather bud and interbud domains arranged in highly ordered arrays. The first step in skin pattern induction is the formation of the dense dermis. In the dorsal skin, dermal cells migrate from the medio-dorsal lip of the somites under the ectoderm (Olivera-Martinez et al., 2000) and extend progressively from the median to the lateral dorsal region. The dermis of the limbs and of the ventral part of the body arises from the somatopleural mesoderm. In the limb, the dense dermis extends in a posterior to anterior direction. During dense dermis formation, no morphological differences between presumptive feathers and interbud regions are detectable. The dense dermis induces the formation of epidermal placodes, the first histological recognisable elements of the feather buds. In turn, the epidermal placode signals to the dermis and induces dermal cells to aggregate (Jiang et al., 1999) to form the dermal condensation which acquires its own inductive properties. Reciprocal interactions between ectoderm and dermis result in patterning and growth of the bud (reviewed in Sengel, 1976; Dhouailly, 1977; Chuong, 1998).
It was previously reported that many of the signalling molecules expressed in the feather bud can either activate the formation of feather primordia, (Shh, FGF, $\beta$-catenin, TGF, Delta-1), or inhibit it (BMP2, BMP4, Notch-2, EGF) (Ting-Berreth and Chuong, 1996; Widelitz et al., 1996; Crowe et al., 1998; Jung et al., 1998; Morgan et al., 1998; Viallet et al., 1998; Noramly et al., 1999; Atit et al., 2003). The feather bud initiation and growth could be regulated by the relative ratios between activators and inhibitors (Jung et al., 1998) and by lateral inhibition processes (Crowe et al., 1998; Noramly and Morgan, 1998; Viallet et al., 1998).

The BMPs are commonly considered as inhibitors of bud formation that contribute to the spacing between initiating buds as well as to the subsequent patterning of each individual feather (Jung et al., 1998; Noramly and Morgan, 1998). Accordingly, increasing the sensitivity to BMPs in dermal cells by overexpressing Bmp Receptors, in a feather reconstitution assay, decreases the size of feather bud (Jiang et al., 1999). Similarly, Bmp overexpression in the ectoderm of the feather field inhibits feather bud formation (Noramly and Morgan, 1998). Reciprocally, decreasing the sensitivity to BMPs by overexpression of the BMP

Abbreviations used in this paper: BMP, bone morphogenetic protein; CEF, chick embryo fibroblasts.

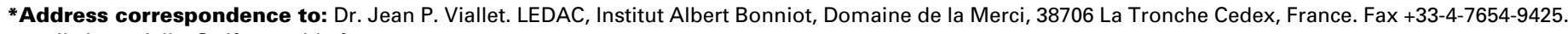
e-mail: Jean.viallet@ujf-grenoble.fr
}

$\boldsymbol{\alpha}$ Present address: Department of Microbiology and Molecular Genetics, Stafford Hall, University of Vermont, Burlington VT05405, U.S.A.

\# Note: Both authors contributed equally to this work.

0214-6282/2004/\$25.00

(C) UBC Press

Printed in Spain

www.ijdb.ehu.es 
antagonist Noggin increases the size of the feather bud at the expense of interbud region (Noramly and Morgan, 1998; Jiang et al., 1999). All these data suggest that BMPs play a crucial role in the lateral inhibition process between adjacent feather primordia. Paradoxically, Bmps are expressed in both the epidermal placode and the dermal condensation as they form (Noramly and Morgan, 1998) raising the question of how feather buds themselves escape the inhibitory actions of BMPs. It has been suggested that Follistatin, a BMP antagonist expressed within feather buds, could inhibit BMP signalling in the feather placode below a particular threshold and this would allow feather formation (Patel et al., 1999). Because Follistatin interacts with extracellular matrix components (Hashimoto et al., 1997), it has been postulated that BMPs would diffuse further than Follistatin and hence they would be able to act in interbud regions to inhibit feather bud formation. Accordingly, application of Follistatin to feather cultures induces feather bud formation in interbud regions (Patel et al., 1999).

We have identified another BMP antagonist, drm (Topol et al., 1997), also termed gremlin (Hsu et al., 1998), whose expression
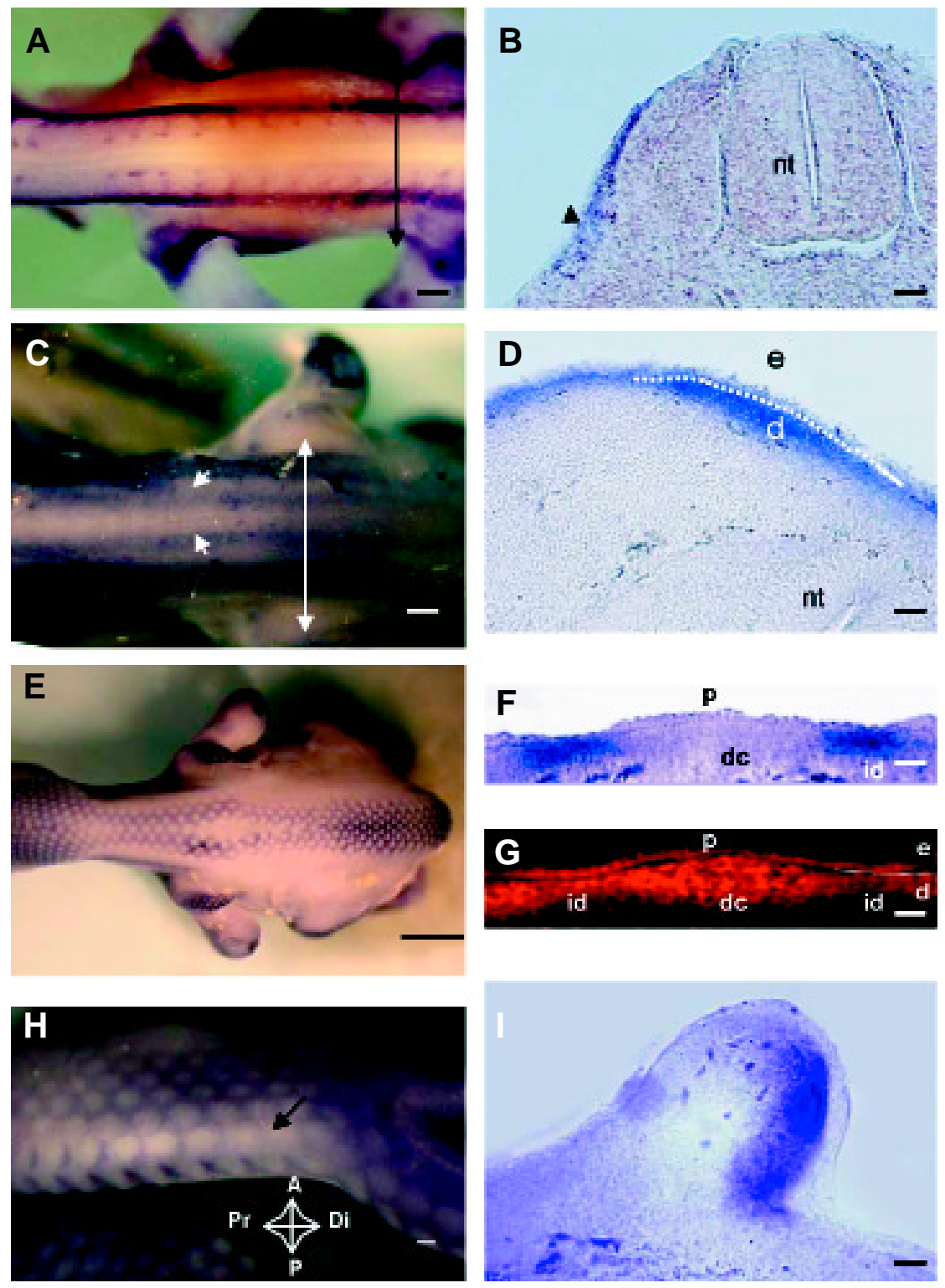

pattern in the developing skin differs significantly from that of follistatin. Drm is a secreted and cell-membrane associated glycoprotein (Topol et al., 2000), belonging to the Dan family of cystine knot secreted proteins (Hsu et al., 1998; Pearce et al., 1999; Piccolo et al., 1999). Drm binds BMP-2 and BMP-4 and controls outgrowth, chondrogenesis and apoptosis during chicken and mouse limb development (Capdevila et al., 1999; Merino et al., 1999; Zuniga et al., 1999). We and others have previously shown that $\mathrm{drm}$ displays a dynamic expression pattern during limb buds, branchial arches, dermatome and feather bud development (Merino et al., 1999; Bardot et al., 2001; Ohyama et al., 2001).

In this report we show that Drm/Gremlin is an activator of feather bud development, since overexpression of mouse $\mathrm{drm}$ results in fusions between adjacent feather buds. We also show that expression of endogenous $d r m$ is under the control of a feedback loop induced by the BMP pathway. Given that $d r m$ is expressed in dermis prior to feather bud induction and in interbud regions during buds formation, our results suggest that endogenous Drm can contribute to the size and spacing of the feather buds control by antagonizing the inhibitory effect of BMPs during feather development.

\section{Results}

\section{Drm/gremlin is expressed in the dermis before feather bud formation}

We have previously reported that during early stages of chicken development $\mathrm{drm} / \mathrm{grem} / \mathrm{in}$ is expressed in the dermomyotome (Bardot et al., 2001). Analysis of expression pattern of $\mathrm{drm} /$ gremlin during feather development from the stage of ectodermal placode formation onwards

Fig. 1. Expression pattern of $\boldsymbol{c d r m}$ during skin and feather development. (A) $26 \mathrm{HH}$ embryonic stage, dorsal view. (B) Transverse section of (A) (indicated by double headed arrow). Arrowhead indicates that drm expressing cells are located in the lateral sub-ectodermal space. (C) $29 H \mathrm{H}$ embryonic stage, dorsal view. Drm is expressed in two broad dorsal stripes indicated by white arrowheads. (D) Transverse section of (C) (indicated by double headed arrow). Endogenous drm transcripts are detected in the dermis (d) and not in the epidermis (e). (E) $32 \mathrm{HH}$ embryonic stage, dorsal view; $\mathrm{drm}$ is expressed in interbud regions. (F,G) Section of (E), drm transcripts are detected in the interbud dermis (id) and not in the feather primordia formed by a placode (p) and a dermal condensation (dc). (G) Propidium iodide counterstain of $F$. (H) $A$ $34 \mathrm{HH}$ wing, showing the three steps of $\mathrm{drm}$ expression: in the most anterior (youngest) buds, drm is expressed around the feather buds; in the last but one row, drm expression is lowered (black arrow), and in the most posterior row, drm is expressed in the posterior half of each feather bud. (I) Transverse section showing that during feather bud outgrowth, drm expression is restricted in posterior dermal cells of the bud. A-C and E, heads to the left. nt, neural tube; Pr-Di, proximo-distal axis; $A-P$, anteroposterior axis. Size bars, $(A, C) 250 \mu \mathrm{m}$; (B,D) $30 \mu \mathrm{m}$; (E) $800 \mu \mathrm{m}$; (F-I) $12.5 \mu \mathrm{m}$. 
(Ohyama et al., 2001) showed that $d r m$ is dynamically expressed during feather morphogenesis. Taken together, these data suggested a possible role of Drm in skin pattern formation.

We first analyzed the expression pattern of $d r m$ from earlier stages, before the onset of the dense dermis formation $(26 \mathrm{HH})$ onwards $(36 \mathrm{HH})$. We showed that at stage $26 \mathrm{HH}, d r m$ is expressed in cells of the subectodermal space (Fig. 1 A,B). At stage $29 \mathrm{HH}$, prior to any morphologically detectable formation of the ectodermal placode, drmis expressed in two broad dorsal stripes (Fig. 1C). Transverse sections show a homogeneous distribution of $d r m$ transcripts in the dermis at this stage (Fig. 1D). From stages $30 \mathrm{HH}$ to $32 \mathrm{HH}$, drm is expressed between feather primordia, and its expression is down regulated in the primordia (Fig. 1E). Transverse sections revealed that its expression is restricted to the dermis of interbud spaces outside of the boundary of the feather primordia (Fig. 1 F,G). At HH34, drmexpression decreased around the more advanced dorsal rows of feather buds (Fig. $1 \mathrm{H})$, whereas at later stages $(36 \mathrm{HH}$ onwards), it is detected only in the posterior half the of the feather bud (Fig. 1I). Interestingly, it is never expressed in apteri.

\section{Overexpression of $\mathrm{drm} / \mathrm{gremlin}$ induces fusion of feather buds}

To analyze the role of Drm in feather morphogenesis, we studied the effect of its overexpression on the formation of feather buds. We therefore grafted aggregates of chicken fibroblasts infected with an RCAS retrovirus expressing the mouse $d r m$, between the neural tube and the somites of E2 chicken embryos. From stage 31 to $36 \mathrm{HH}$ two major but not mutually exclusive modifications of feather phenotype are observed in dorsal areas expressing mouse $\mathrm{drm}$. In some cases $(n=12)$, several feather buds are larger than normal (Fig. 2 F,G arrowheads). But in most cases $(n=26)$, fusion of adjacent feather buds is observed (Fig. $2 \mathrm{~F}$ arrows). Finally, at stage $38 \mathrm{HH}$, the fused buds generated feathers fused all over, except at the most distal tip (Fig. 2H), whereas the enlarged buds generated feathers resembling baseball bats (Fig. 2l).

To correlate the observed phenotypes with the expression of $m d r m$, grafted embryos were fixed at different stages and analyzed by whole mount in situ hybridization. Transverse sections of the embryos at the grafted region showed the progression of the transgene expression before and during the onset of feather bud formation. At stage $22 \mathrm{HH}, m d r m$ transcripts are co-detected

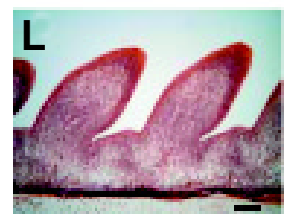

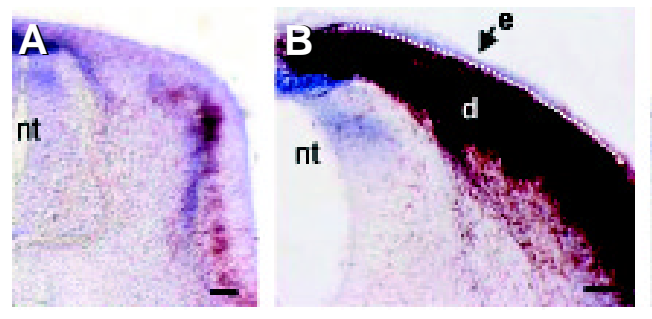
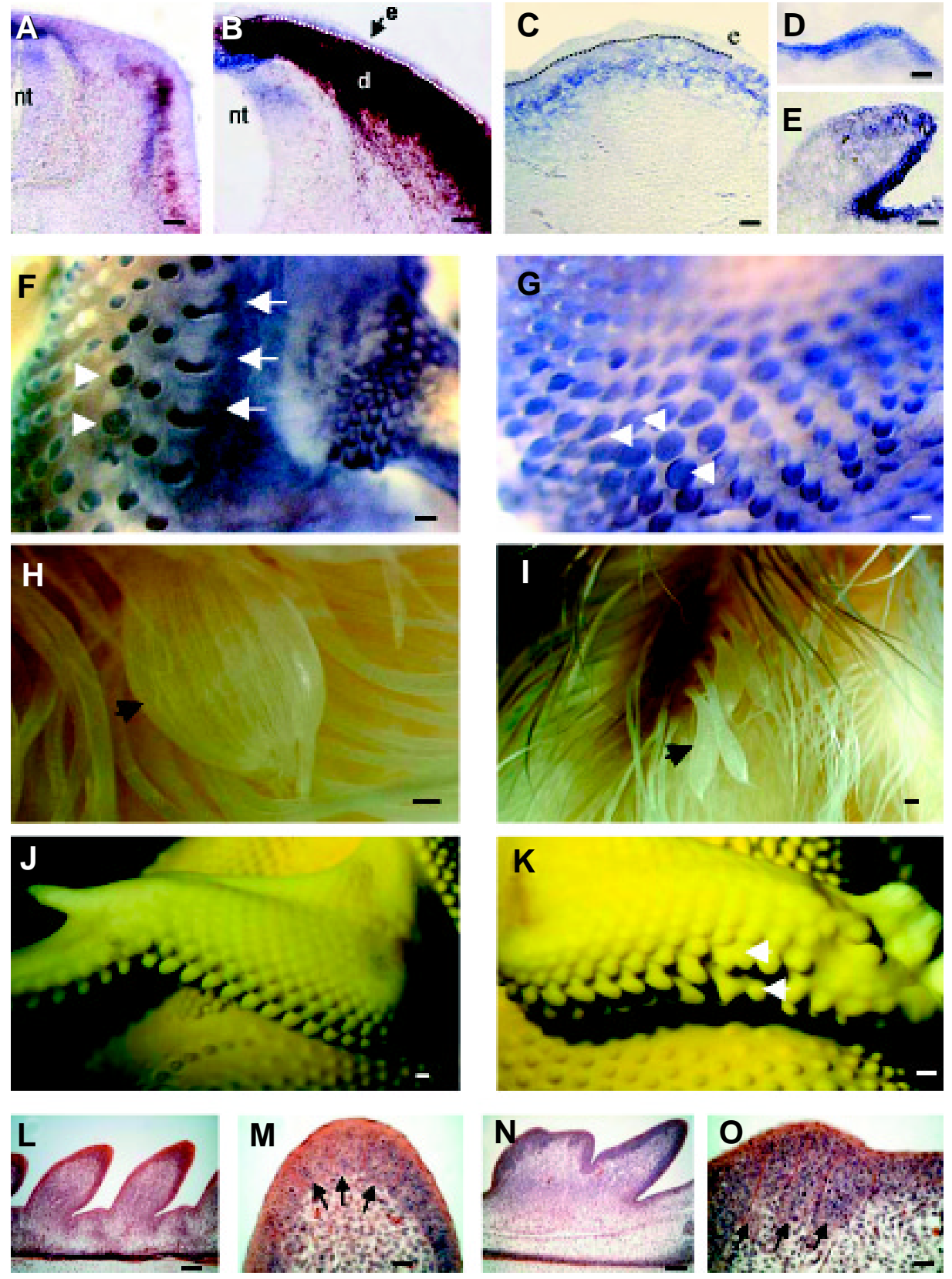

Fig. 2. Overexpression of $\mathbf{d r m}$ alters feather formation. (A-G) Embryos grafted with RCAS/mdrminfected fibroblasts between neural tube and somites harvested at stage $22 \mathrm{HH}(\mathbf{A}), 24 \mathrm{HH}(\mathbf{B}), 31 \mathrm{HH}$ (C), $34 \mathrm{HH}$ (D), 36HH (E,F,G) and hybridized either with an mdrm antisens riboprobe alone (blue) $(C, D, E, F, G)$ or with both mdrm (red) and c-hairy1 (blue) antisens riboprobes (A,B). The fusions between adjacent feather buds located on adjacent rows $(\mathbf{F}, \mathbf{H})$ are indicated by arrows. The enlarged feather buds $(F, G)$ are indicated by white arrowheads. The fused feathers $\mathbf{( H )}$ and the feathers with a baseball bats-like aspect (I) are indicated by black arrows. (J,K) Embryos grafted in wing buds and harvested at stage 36HH. (J) Non-infected, control left wing. (K) RCAS/mdrm-infected right wing with fused feathers (white arrows). Section of normal feather buds in the control wing (L,M) and of

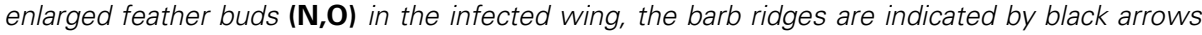
(Hematoxylin Biebritch Scarlet staining). nt, neural tube; e, ectoderm; d, dermis. Size bars, (A-E) 30 um; (F-I) $150 \mu \mathrm{m} ;(\mathrm{J}, \mathrm{K}) 200 \mu \mathrm{m}$; (L,M) $100 \mu \mathrm{m}$; (N, O) $50 \mu \mathrm{m}$.

with $c$-hairy 1 in the mediodorsal lip of the dermomyotome (Fig. $2 \mathrm{~A}$ ). At stages $22 \mathrm{HH}$ to $24 \mathrm{HH}$, they rapidly spread (Fig. $2 \mathrm{~B}$ ) and then occupy the whole dermis (Fig. $2 \mathrm{C}$ ). At stage $34 \mathrm{HH}$, after the primordia formation, transcripts extend to the epidermal cells 
during the feather bud outgrowth (Fig. 2D,E). Surprisingly, at later stages (HH36) (Fig. 2F), the transgene is mainly expressed in the developing feathers. It is only slightly detectable in the deep interfeather dermis. At this stage, its expression extends over a large part of the dorsal region. Interestingly, not all the feathers within
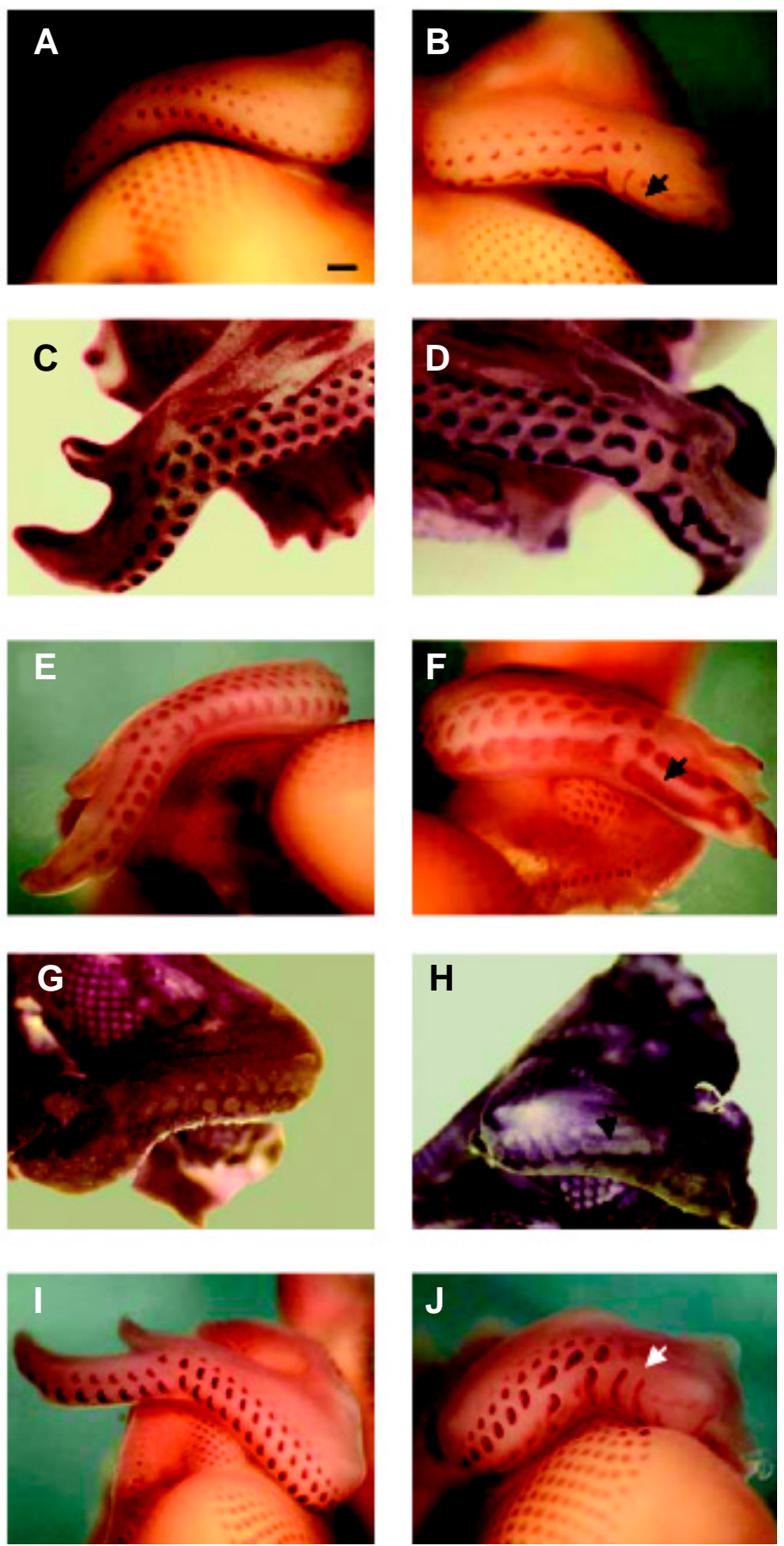

Fig. 3. Expression pattern of feather bud developmental markers in Drm-induced fused feathers. E4 embryos grafted in the right wing bud were harvested four days later. $(A, C, E, G, I)$ Left non-infected wing; $(B, D, F, H, J)$ Right infected wing, hybridized with antisens riboprobes forshh (A,B), Bmp4 (C,D), Bmp2 (E,F), Notch2 (G,H) and delta-1 (I,J). Black arrowheads indicate fusion between feather buds of the same row. White arrowhead indicates fusion between feather buds of adjacent rows. Size bar, (A-J) $100 \mu \mathrm{m}$. the infected area display a phenotype and no ectopic feathers were observed within normally apteric areas (Fig. 2F). Moreover, $d r m$ missexpression has no noticeable effect on the anteroposterior orientation of the feathers. This is shown by the fact that in embryos overexpressing $d r m$, normal, enlarged and even fused feathers exhibit a normal antero-posterior orientation at all stages examined. Grafts of fibroblasts infected with empty vector $(n=16)$ never induced abnormal feathers formation or disorganization of feather tracts.

The histological analysis showed that enlarged fused feathers include a larger dermal condensation than the normal feather bud, while the dermal cell density is comparable to the normal feather (Fig. 2 L-O). Interestingly normal barb ridges form from the epidermis of the fused feather buds (Fig. 2 N,O). These observations suggest that $\mathrm{drm}$ overexpression induced both epidermis and dermis to adopt a feather fate within an area normally endowed with an interfeather fate.

In order to determine whether phenotypes induced by $\mathrm{drm}$ overexpression resulted from a specific dermatome perturbation, or occurred during feather primordia formation, we grafted RCAS/ mdrm-infected fibroblasts in the posterior part of the wing buds of stage $21 \mathrm{HH}$ embryos and the phenotypes were analyzed 6 days later. In this case, although feather bud fusions are observed, in the majority of embryos they occurred between primordia of the same row (Fig. 3 B,D,F,H). In some cases, fusions between adjacent primordia of different rows as in the dorsal pterilae are also observed (Fig. 3J). These results clearly showed that the overexpression of $\mathrm{drm}$ affects mechanisms involved in feather primordia formation rather than development of the dermatome.

\section{Effect of drm/gremlin overexpression on the expression of feather bud markers}

The phenotypes induced by $d r m$ overexpression could be either the cause or the consequence of transcriptional modifications of genes expressed during feather bud development. To test this possibility we analyzed the expression pattern of several genes at stage $34 \mathrm{HH}$ in wings grafted with mdrm-expressing fibroblasts. The presence of fused feather primordia and digit malformations in the grafted wings were positive controls for efficient expression of the transgene (Fig. 3 B,D,F,H and J). The opposite non-grafted wing was used as a control for the normal gene expression pattern (Fig. 3 A,C,E,G, and I). We show that the expression of Bmp2, Bmp4, shh and delta1, that are normally restricted within the primordia are ectopically induced in the fused feathers in the grafted wings, and that notch2 normally expressed in the interbud dermis, is down regulated in these fused feathers (Fig. $3 \mathrm{~B}, \mathrm{D}, \mathrm{F}, \mathrm{J}$ and $\mathrm{H}$ respectively). However, no modification of the expression of these genes is observed in the normal feathers within the infected wings. Thus, the distribution of the Bmp2, Bmp4, shh, delta1 and Notch2transcripts in the dermis and the epidermis of the fused or enlarged primordia, is simply the consequence of the phenotype induced and does not reflect specific up or down regulation of these genes by $m d r m$. Identical results were obtained in the dorsal pteryla of embryos grafted between neural tube and somites (data not shown). These data suggest that feather phenotype induced by mdrmoverexpression was the consequence of the antagonistic effect of Drm on BMP signaling during feather primordia formation rather than of a direct effect on the expression of feather bud markers. 


\section{Expression of endogenous drm/gremlin in feather buds is under the control of BMP signaling}

The observation that in feather buds, the expression pattern of $d r m$ is always complementary to that of Bmp2and Bmp4, suggests that BMP signaling could regulate endogenous expression of $\mathrm{drm}$. We therefore analyzed the expression pattern of endogenous $\mathrm{drm}$ following RCAS-driven Bmp4 missexpression in developing skin. It has been previously reported that overexpression of $B m p 2$ and $B m p 4$ induced large apterous areas in chicken skin and that this phenotype resulted from the inhibition of feather bud development at a stage after dense dermis formation but prior to placode specification (Noramly and Morgan, 1998). We grafted RCAS/ Bmp4-infected fibroblasts into E2 embryos $(\mathrm{HH} 11-15)$ in the space between neural tube and somite at the trunk level, and analyzed the skin morphology and endogenous drmexpression at E8 (HH 34). All of the grafted embryos $(n=11)$ display the glabrous phenotype previously described. At the level of the graft, BMP4 overexpression mainly induced large glabrous areas that extend over a large part of the dorsal skin (Fig. 4 A,B). Small apteric areas located either at the level of the graft (Fig. 4 C,D), or distantly from it are also observed, the later probably resulting from secondary infection events, since BMP4 transcripts are expressed independently of the main infection area (data not shown).

Expression pattern of endogenous $d r m$ within and around glabrous fields was also affected. Within large areas devoid of feather buds, a low expression of endogenous drm is detected, whereas $d r m$ expression is strongly enhanced at the periphery of these apterous areas (Fig. 4 A,B). On the contrary, within small apteric areas, expression of endogenous drm is activated (Fig. 4 C,D). Identical apterous phenotypes and modifications of the endogenous $\mathrm{drm}$ expression were obtained following overexpression of a constitutively activated form of the BMPR1A (Fig. 4 E,F). Interestingly, in some cases we observed the appearance of fused feather buds at the periphery of large apteric areas induced by BMPR1A or BMP4 overexpression (see black arrowhead Fig. 4E and data not shown). All these results suggest that in developing skin, the expression of $d r m$ should be under the control of BMP signaling.

\section{Discussion}

The dynamic expression pattern of $d r m$ / gremlin, together with the effects of its misexpression raises several hypotheses about its roles during avian skin morphogenesis. The experimental data presented above suggest that Drm is a dermal factor involved in feather tract patterning, primordia formation and bud morphogenesis.

\section{Drm/gremlin expression in dermal cells is complementary to that of BMPs}

The $\mathrm{drm} /$ gremlingene is expressed in dermal cells from early stages of dermal precursor formation in the somite (Bardot et al., 2001; and this work) to dense dermis and feather pattern formation. This observation suggests that $\mathrm{drm} / \mathrm{grem} / \mathrm{in}$ is one of the earliest genes expressed in the dermis along with the bHLH transcription factor Dermo-1 (Scaal et al., 2002). However, after feather placode formation Dermo-1 is principally expressed in the dermal condensations, whereas the expression of $d r m$ is restricted to the interbud dermis. At later stages, $d r m$ is expressed by dermal cells
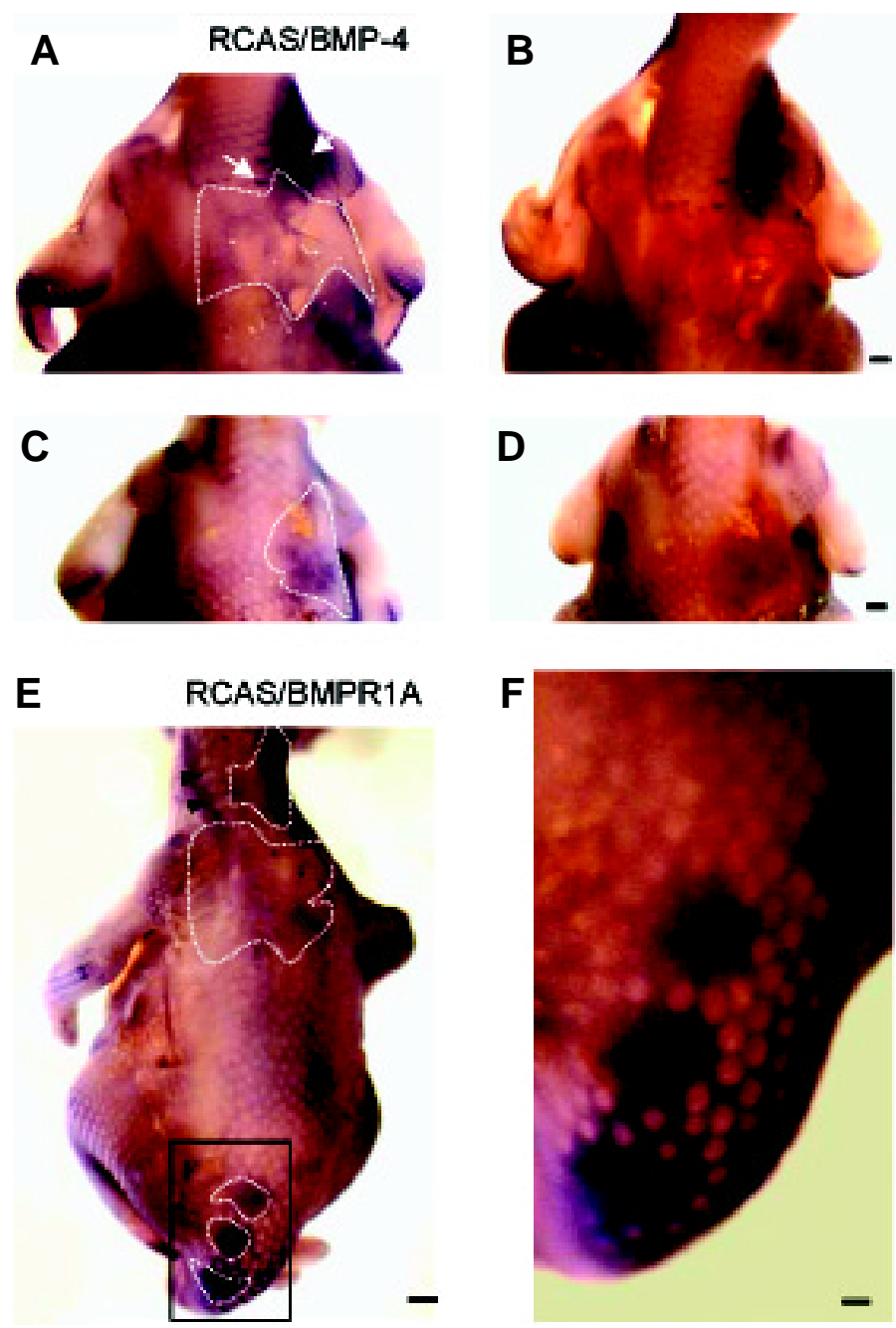

Fig. 4. Expression of endogenous drm is under the control of BMP signaling. Embryos grafted with RCAS/BMP4 (A-D) and with RCAS/ hBMPR1A-infected-cells (E,F) were harvested at stage $32 \mathrm{HH}$. They were hybridized with a cdrm antisense riboprobe (blue staining in $A, C, E, F)$ and with a BMP4 antisense probe (red staining in $B, D$ ). (A,B) Embryo displaying large apterous areas. The center of the large apterous area displays low endogenous drm expression, while at the periphery of the infected region, cdrm expression is strongly enhanced (white arrowhead). (C,D) Embryo displaying small apterous areas. (E,F) Large and small glabrous areas induced by overexpression of an activated form of BMPR1A. Black arrowheads indicate fused feather buds. Square in (E) indicates small secondary apterias illustrated in (F). (F) These apteriae exhibit upregulation of endogenous drm expression. Size bars, (A-E) $700 \mu \mathrm{m}$; (F) $200 \mu \mathrm{m}$.

recruited in the posterior part of the forming feather. It is noteworthy that at all stages, $d r m /$ gremlinexpression field is complementary to those of $b m p-2$ and $b m p-4$ previously described (Jung et al., 1998; Noramly and Morgan, 1998). Bmp-2 is first expressed in a large stripe of ectoderm that covers the dense dermis and this expression is progressively restricted to the ectodermal placode when the feather primordia form. Both $b m p-2$ and $b m p-4$ are expressed in the nascent dermal condensation. During feather bud outgrowth and orientation bmp expression is restricted to the anterior region of the bud in both the ectoderm and the dermis. As Drm/Gremlin binds BMP-2 and BMP-4, two signaling molecules 
known to inhibit feather commitment at successive stages in feather tract development, the exclusive patterns of $\mathrm{drm}$ and bmps suggest reciprocal roles for these molecules.

\section{BMPs regulate drm/gremlin expression}

It was recently shown that expression of $d r m$ is under the control of BMP signaling in osteoblasts (Pereira etal., 2000) as well as into chick limb buds. In this case, BMP-soaked beads decreased $\mathrm{drm}$ expression in a region close to the BMP source, whereas upregulation occurred at a distance (Capdevila et al., 1999; Merino et al., 1999).

The BMPs overexpression elicits different effects according to the skin developmental stage: at early stages $(17-23 \mathrm{HH})$ implantation of beads soaked with BMPs can induce ectopic feather formation in a particular limited region of the lateral trunk (Scaal et al., 2002) while all over and at later stage (29 HH) BMPs inhibit feather formation (Jung et al., 1998; Noramly and Morgan, 1998). In our hand, the overexpression of either BMP4 or of constitutively activated form of the BMPR1A leads to the formation of larges and secondary small apterics regions. The $d r m$ expression is enhanced at the periphery of the large apteric areas and in the small glabrous regions. This effect of bmp4 overexpression on endogenous $d r m$ in the BMP-induced apteric regions suggests that, in developing skin, expression of $d r m$ is under the control of BMP signaling.

However, the positive regulation model of $d r m$ expression by the BMP signaling can not explain the low expression level of $\mathrm{drm}$ within the large BMP-induced apteric areas. We can speculate that in skin expression of $d r m$ could be regulated by a threshold effect of BMP on BMP signaling as it has been proposed during early limb bud development (Capdevila et al., 1999; Merino et al., 1999). Alternatively, expression of $d r m$ could be under the control of additional positive regulatory signals that activate or maintain $\mathrm{drm}$ expression at the periphery of apteric regions. These signals could be provided from surrounding feather buds that are located too far to activate $d r m$ expression in the center of large glabrous areas. It is noteworthy than during limb bud development Shh and FGF pathways are required for maintenance of $\mathrm{drm} /$ gremlinexpression (Merino et al., 1999; Zuniga et al., 1999).

\section{Drm/gremlin regulates feather primordia formation}

The overexpression of $d r m$ leads to the formation of enlarged and fused primordia suggesting that Drm is a crucial and limiting factor in the early steps of feather induction. The progression in time of the infection is important in order to interpret the $\mathrm{drm}$ phenotype. The graft of RCAS marm-producing cells between the neural tube and the somites leads to a strong expression of $m d r m$ at early stage $\left(\mathrm{HH}_{2} 4\right)$ that remains restricted to the dermal cells until feather bud outgrowth. Interestingly, overexpression of $m d r m$ at early stage did not modify the dense dermis formation. These results suggest that, in contrast to bHLH transcription factor Dermo-1 (Scaal et al., 2002) and the cell-cell adhesion molecule NCAM (Jiang et al., 1999) both recently showed to be involved in this process, the homogeneous expression of endogenous drmat this stage is not the limiting factor for dense dermis extension. Later, $d r m / g r e m l i n$ overexpression led to the fusion of laterally adjacent feather buds. This phenotype seems to be the consequence of the homogenous expression of $d r m$ in the dermis at the time of primordia formation, rather than of the ectopic expression of $m d r m$ in the epidermis during later feather bud outgrowth. This is supported by the fact that the progression of the infection from the dermis to the ectoderm occurred after the modification of expression of early markers and the formation of fused primordia. Moreover, the expression of the feather bud markers ( Shh, Bmps and Delta 1) was enlarged in fused primordia but no modification of expression was observed in regions outside of the feather field. This suggests that the feather phenotype induced by $\mathrm{drm}$ overexpression is the consequence of the antagonistic effect of Drm on BMP signaling rather than an indirect effect of Drm on the expression of these signaling molecules. Interestingly in embryos infected with BMP-4 and BMPR $1 \alpha$ expressing retroviruses, large apteric areas are surrounded in some cases by some fused feather buds. This phenotype is always associated with an enhancement of endogenous $\mathrm{drm} / \mathrm{grem}$ linexpression at the periphery of the large apteric areas. This phenotype could result from the up-regulation of expression of endogenous $d r m$ in these regions. On the contrary, within small apteric areas, though expression of endogenous $\mathrm{drm}$ is activated, the skin is always glabrous. This indicates that in these cases, enhancement of endogenous $\mathrm{drm}$ expression could not overcome the interbud fate induced by activation of BMP signalling.

Because of the sequence of feather formation in the tract, the bud fuses with the "younger" of equidistant primordia. The pattern of fusion suggests that the interbud region becomes resistant to primordia formation in a brief period of time between the formation of adjacent buds. During a short time window in bud initiation, drm expressed in the interbud spaces could regulate the size of feather buds and/or the size of interbud regions by interacting with BMPs and inhibiting their signaling, thus preventing early commitment of cells to an interbud fate. Overexpression of $d r m$ in the dermis prior this short period of time would allow both epidermis and dermis to adopt a feather fate within an area normally endowed with an interfeather fate by antagonizing the BMPs. Finally, it was recently reported that at early stage of feather morphogenesis, EGF signaling is required for the determination of interbud fate (Atit et al., 2003). The inhibition of EGFR signaling in skin explants led to the induction of phenotypes similar to those induced by missexpression of $m d r m$, namely to the loss of interbud fate and the feather bud fusion. Further experiments should clarify the links between EGF and $d r m$ in skin.

\section{Feather bud formation and BMP antagonists}

Both $\mathrm{drm} /$ gremlinand follistatinare the only BMP antagonists currently known to be endogenously expressed during feather morphogenesis. It has been previously demonstrated that expression of follistatin is also dependent on BMP activity (Patel et al., 1999). During feather bud development, follistatin is coexpressed with bmps (Patel et al., 1999) and FGFs, whereas $d r m$ exhibits an expression pattern complementary to that of bmps. This suggests that their expression could be regulated by different levels of BMP activity. Follistatin overexpression in the skin leads to the formation of ectopic feathers (Patel et al., 1999). We never observed ectopic formation of primordia after $\mathrm{drm}$ overexpression. Taken together these results suggest that these proteins have different functions during feather development. Follistatin could inhibit BMPs activity within the primordia, and by promoting FGF activity, controls feather bud outgrowth. We propose that BMPs induce $d r m$ expression in the dermis around 
the feather bud. In turn, Drm protein could regulate the size of interbud region by limiting the concentration of available BMPs. This inhibition could allow feather bud induction at a defined distance from the preceding row, by preventing early commitment of the dermal cells to form an apteric region.

\section{Material and Methods}

\section{Preparation and grafting of retrovirus-producing CEF}

Primary chick embryo fibroblasts (CEF) prepared from 11 days old Oline embryos (BBSRC; Institute for Animal Health, Compton, Bercshire, UK) were transfected with RCAS (BP)/mdrm, RCAS/mBmp4, RCAS/ $h B m p R 1 A$ constructs or with RCAS (BP) plasmid using the"Effectene transfection reagent" (Qiagen). The day before grafting, infected cells were plated at high density on bacterial dishes to induce formation of cell aggregates. Those of approximately $100 \mu \mathrm{m}$ were grafted into Brown Leghorn chicken embryos of the Edinburgh strain, either at 11 to 15 Hamburger-Hamilton $(\mathrm{HH})$ developmental stages (Hamburger and Hamilton, 1951), between neural tube and somites at the level of somites 10 to 29 , or in the anterior edge of the right forelimb at stages $21 \mathrm{HH}$. After further development occurred, embryos were fixed in 4\% formaldehyde 2 mM EGTA and analysed by whole-mount in situ hybridization.

\section{Whole-mount in situ hybridization}

The hybridization was performed as described (Henrique et al., 1995). The proteinase $\mathrm{K}$ treatment varied from 10 to $20 \mu \mathrm{g} / \mathrm{ml}$ for one hour, depending on the developmental stage of the embryo. Simultaneous detection of endogenous and retroviral transcripts using two colours in situ hybridization was achieved as previously described (Delfini et al., 2000). Reagents were purchased from Roche. The stained embryos were processed for cryosections following inclusion in gelatin/sucrose.

\section{Plasmids}

$\mathrm{RCAS} / m d r m$ and $\mathrm{pBKS} / m d r m$ constructs were obtained by inserting a 1202bp Xbal fragment containing the coding region and $5^{\prime}$ and $3^{\prime}$ untranslated sequences of mouse drmcDNA (Zhang et al., 2000) into the $\mathrm{Cla}$ /and $\mathrm{Xba}$ /sites of RCAS and Bluescript (Stratagene) vectors respectively. mdrm cDNA was kindly provided by D. Blair. RCAS/mBMP4 was kindly provided by D. Duprez. RCAS/hBMPR1Awas a gift of L. Niswander. The constructs used to generate anti-sense riboprobes $\mathrm{pBKS} / C B M P 2$ and $\mathrm{pBKS} / c B M P 4$ were kindly provided by A.H. Monsoro-Burq, $\mathrm{pBKS} / c^{-}$ hairy 1 by I. Palmeirim, pBKS/cDelta 1 by D. Henrique, pBKS/cNotch2, by R. Goitsuka, pBKS/cShh (Riddle et al., 1993) was generated by RT-PCR and pCR2.1/cdrm was previously described (Bardot et al., 2001).

\section{Acknowledgements}

We thank Dr. D. Duprez, Dr. L. Niswander for virus, Dr. A. HMonsoroBurq, Dr. D. Henrique, Dr Palmeirin, Dr. R. Goitsuka, Dr. D. Blair for constructs, Pr. M. Block for support, Pr. D. Dhouailly for support and critical reading of the manuscript, Pr. S. Nonchev for comments on the manuscript and Dr. D. Pearton and Dr. J. Thelu for advice, B. Peyrusse for iconography and G. Chevalier for technical assistance. B. Bardot, I. Fliniaux and $E$. Huillard were the recipients of doctoral fellowships from the French Ministere de l'Education Nationale, de la Recherche et de la Technologie. I. Fliniaux was also the recipient of a fellowship from the E.P.H.E. B. Bardot was also supported by fellowships from the Ligue Nationale contre le Cancer and the Association pour la Recherche sur le Cancer. This work was supported by the Centre National de la Recherche Scientifique (CNRS) and the Institut Curie and by a grant from IAB to J.P. Viallet.

\section{References}

ATIT R, CONLON RA, NISWANDER L. (2003). EGF signaling patterns the feather array by promoting the interbud fate. Dev Cel/4: 231-240.
BARDOT B, LECOIN L, HUILLARD E, CALOTHY G, MARX M. (2001). Expression pattern of the $\mathrm{drm} / \mathrm{grem}$ lin gene during chicken embryonic development. Mech Dev 101:263-265

CAPDEVILA J, TSUKUI T, RODRIQUEZ ESTEBAN C, ZAPPAVIGNA V, IZPISUA BELMONTE JC. (1999). Control of vertebrate limb outgrowth by the proximal factor Meis2 and distal antagonism of BMPs by Gremlin. Mo/ Ce//4: 839-849.

CHUONG CM, R.B. (1998). Feather morphogenesis: a model of the formation of epithelial appendages. In Molecular Basis of epithelial appendage morpho genesis. (Ed. Chuong CM). Georgetown, Texas: R.G. Landes Co. p 57-74.

CROWE R, HENRIQUE D, ISH-HOROWICZ D, NISWANDER L. (1998). A new role for Notch and Delta in cell fate decisions: patterning the feather array. Development 125: 767-775.

DELFINI M, HIRSINGER E, POURQUIE O, DUPREZ D (2000). Delta 1-activated notch inhibits muscle differentiation without affecting Myf5 and Pax3 expression in chick limb myogenesis. Development 127:5213-24.

DHOUAILLY D. (1977). Dermo-epidermal interactions during morphogenesis of cutaneous appendages in amniotes. in: Robert, I., (Ed.), Frontier Matrix Biology. Creteil vol. 4. pp.86-121

HAMBURGER V, HAMILTON,H.L. (1951). A series of normal stages in the development of the chick embryo. J.Exp.Morphol. 88: 49-92.

HASHIMOTO O, NAKAMURA T, SHOJI H, SHIMASAKIS, HAYASHIY, SUGINO $H$. (1997). A novel role of follistatin, an activin-binding protein, in the inhibition of activin action in rat pituitary cells. Endocytotic degradation of activin and its acceleration by follistatin associated with cell-surface heparan sulfate. Biol Chem 272: 13835-13842.

HENRIQUE D, ADAM J, MYAT A, CHITNIS A, LEWIS J, ISH-HOROWICZ D (1995). Expression of a Delta homologue in prospective neurons in the chick. Nature 375: 787-790.

HSU DR, ECONOMIDES AN, WANG X, EIMON PM, HARLAND RM. (1998). The Xenopus dorsalizing factor Gremlin identifies a novel family of secreted proteins that antagonize BMP activities. Mol Ce//1: 673-683.

JIANG TX, JUNG HS, WIDELITZ RB, CHUONG CM. (1999). Self-organization of periodic patterns by dissociated feather mesenchymal cells and the regulation of size, number and spacing of primordia. Development 126: 4997-5009.

JUNG HS, FRANCIS-WEST PH, WIDELITZ RB, JIANG TX, TING-BERRETH S, TICKLE C, WOLPERT L, CHUONG CM. (1998). Local inhibitory action of BMPs and their relationships with activators in feather formation: implications for periodic patterning. Dev Biol 196: 11-23.

MERINO R, RODRIGUEZ-LEON J, MACIAS D, GANAN Y, ECONOMIDES AN HURLE JM. (1999). The BMP antagonist Gremlin regulates outgrowth, chondrogenesis and programmed cell death in the developing limb. Development 126: 5515-5522.

MORGAN BA, ORKIN RW, NORAMLY S, PEREZ A. (1998). Stage-specific effects of sonic hedgehog expression in the epidermis. Dev. Biol. 201: 1-12.

NORAMLY S, FREEMAN A, MORGAN BA. (1999). beta-catenin signaling can initiate feather bud development. Development 126: 3509-3521.

NORAMLY S, MORGAN BA. (1998). BMPs mediate lateral inhibition at successive stages in feather tract development. Development 125: 3775-3787.

OHYAMA A, SAITO F, OHUCHI H, NOJIS. (2001). Differential expression of two BMP antagonists, gremlin and Follistatin, during development of the chick feather bud. Mech Dev 100: 331-333.

OLIVERA-MARTINEZ I, COLTEY M, DHOUAILLY D, POURQUIE O. (2000) Mediolateral somitic origin of ribs and dermis determined by quail-chick chimeras. Development 127: 4611-4617.

PATEL K, MAKARENKOVA H, JUNG HS. (1999). The role of long range, local and direct signalling molecules during chick feather bud development involv ing the BMPs, follistatin and the Eph receptor tyrosine kinase Eph-A4. Mechanisms of Development 86: 51-62.

PEARCE JJ, PENNY G, ROSSANT J. (1999). A mouse cerberus/Dan-related gene family. Dev Bio/209: 98-110.

PEREIRA RC, ECONOMIDES AN, CANALIS E. (2000). Bone morphogenetic proteins induce gremlin, a protein that limits their activity in osteoblasts. Endocrinology 141: 4558-4563

PICCOLOS, AGIUSE, LEYNSL, BHATTACHARYYAS, GRUNZH, BOUWMEESTER T, DE ROBERTIS EM. (1999). The head inducer Cerberus is a multifunctional antagonist of Nodal, BMP and Wnt signals. Nature 397: 707-710. 
RIDDLE R, JOHNSON, R. L., LAUFER, E.AND TABIN, C. (1993). Sonic hedgehogmediatesthe polarizing activityof the ZPA. Cel/75: 1401-1416.

SCAAL M, PROLS F, FUCHTBAUER EM, PATEL K, HORNIK C, KOHLER T, CHRIST B, BRAND-SABERI B. (2002). BMPs induce dermal markers and ectopic feather tracts. Mech Dev 110: 51-60.

SENGEL P. (1976). Morphogenesis of skin. Cambridge: Cambridge Univ. Press.

TING-BERRETH SA, CHUONG CM. (1996). Sonic Hedgehog in feather morphogenesis: induction of mesenchymal condensation and association with cell death. Dev Dyn 207: 157-170.

TOPOL LZ, BARDOT B, ZHANG Q, RESAU J, HUILLARD E, MARX M, CALOTHY G, BLAIR DG. (2000). Biosynthesis, post-translation modification, and functional characterization of Drm/Gremlin. J Biol Chem 275: 8785-8793.

TOPOL LZ, MARX M, LAUGIER D, BOGDANOVA NN, BOUBNOV NV, CLAUSEN PA, CALOTHY G, BLAIR DG. (1997). Identification of drm, a novel gene whose expression is suppressed in transformed cells and which can inhibit growth of normal but not transformed cells in culture. Mo/ Cel/ Bio/17: 4801-4810.

VIALLET JP, PRIN F, OLIVERA-MARTINEZ I, HIRSINGER E, POURQUIE O, DHOUAILLY D. (1998). Chick Delta-1 gene expression and the formation of the feather primordia. Mech Dev72: 159-168.

WIDELITZ RB, JIANG TX, NOVEEN A, CHEN CW, CHUONG CM. (1996). FGF induces new feather buds from developing avian skin. $J$ Invest Dermato/107: 797-803.

ZHANG Q, TOPOL LZ, ATHANASIOU M, COPELAND NG, GILBERT DJ, JENKINS NA, BLAIR DG. (2000). Cloning of the murine Drm gene (Cktsf1b1) and characterization of its oncogene suppressible promoter. Cytogenetics \& Cell Genetics 89: 242-251.

ZUNIGA A, HARAMIS AP, MCMAHON AP, ZELLER R. 1999. Signal relay by BMP antagonism controls the SHH/FGF4 feedback loop in vertebrate limb buds. Nature 401: 598-602. 